\title{
How Different are the Supermanifolds of Rogers and DeWitt?
}

\author{
Jeffrey M. Rabin ${ }^{1 \star}$ and Louis Crane ${ }^{2 \star \star}$ \\ 1 The Enrico Fermi Institute of the University of Chicago, Chicago, IL 60637, USA \\ 2 Department of Mathematics, University of Chicago, Chicago, IL 60637, USA
}

\begin{abstract}
A DeWitt supermanifold always has the structure of a vector bundle over an ordinary spacetime manifold, whereas a Rogers supermanifold is not so restricted. Corresponding to the vector space fibers of the DeWitt supermanifold, a Rogers supermanifold has a foliation by submanifolds, or leaves, parametrized by soul coordinates only. We show that the universal covering space of any leaf always admits a flat metric. If the covering space is complete in this metric, it must in fact be a vector space. We combine this result with known theorems about foliations to give conditions under which a compact Rogers supermanifold with a single even dimension is necessarily a quotient space of flat superspace. We also show that a supermanifold defined by a polynomial equation in flat superspace is always of the DeWitt type. Finally, we exhibit new supermanifold structures for $R^{2}$ and the 2-torus which show that the foliation of a Rogers supermanifold can be quite exotic.
\end{abstract}

\section{Introduction}

Just as general relativity is best formulated in terms of the differential geometry of a four-dimensional spacetime manifold, so the mathematical structure of supergravity is best understood as the differential geometry of a "supermanifold" having both commuting and anticommuting coordinates. Although the supergravity literature focuses on local geometry, rigorous mathematical definitions of supermanifolds have been given which permit the study of global topological questions as well. Of these, the most general definition is that of Rogers [1]. A Rogers supermanifold possesses only the minimal structure needed to define superfields with the properties required in supersymmetric theories. DeWitt's definition [2] is more restrictive, but still adequate for physical applications.

\footnotetext{
* Enrico Fermi Fellow. Research supported by the NSF: PHY 83-01221, and the Department of Energy: DE AC02-82-ER-40073

$\star \star$ Address after September 1, 1985: Institute for Advanced Study, Princeton, NJ 08540, USA
} 
For our purposes a DeWitt supermanifold may be viewed as a Rogers supermanifold with additional structure: there must be a real submanifold, called the body, which can serve as the physical spacetime manifold, and the supermanifold must be a vector bundle over its body. (Actually what we refer to as a DeWitt supermanifold would be called its skeleton in [2], but the distinction will not be important.) In principle a Rogers supermanifold is much more general than a DeWitt supermanifold, but few examples exist which demonstrate this supposed generality. Most published examples of Rogers supermanifolds are either DeWitt supermanifolds or simple quotient spaces thereof. The only exceptions we are aware of are some examples constructed in [3].

Recently it was shown that much of the structure of a DeWitt supermanifold carries over to a Rogers supermanifold [4, 5]. A Rogers supermanifold has a foliation, called the soul foliation, whose leaves correspond to the vector spaces fibering a DeWitt supermanifold. There is also an extensive network of additional foliations corresponding to subspaces of the DeWitt vector spaces. (Recall that a foliation is a smooth slicing of a manifold into submanifolds of a given dimension, called leaves, such that each point of the manifold lies in a unique leaf. The fibers of a fiber bundle are examples of leaves, but foliations can be much more complicated.) In this paper we continue our study of the structure of supermanifolds, pointing out further similarities between the leaves of Rogers supermanifolds and the vector space fibers of DeWitt supermanifolds. Our central result is that the universal covering space of any leaf (or the leaf itself if it is simply connected) always admits a flat Riemannian metric. If the covering space is complete in this metric, it can only be the vector space $R^{k}$ for some $k$. However, even a foliation whose leaves are known to be $R^{k}$ need not be a vector bundle. In Sect. 6 we give an example of a simply connected supermanifold foliated by leaves which are topologically $R^{1}$ but are not fibers of a bundle. The example works because the leaves are not complete in their natural flat metrics. We do not know whether demanding completeness suffices to obtain a vector bundle.

Most of the few general structure theorems for foliations deal with foliations of codimension 1. They therefore have implications for the structure of supermanifolds having a single even dimension. We will show, for example, that a compact Rogers supermanifold with a single even coordinate and a nice enough foliation can only be a quotient space of flat superspace. We also show that most of the complexity of the foliation structure of a supermanifold is associated with the soul foliation: the subsidiary foliations are intrinsically better behaved.

Our other results deal with particular examples of supermanifolds. For real and complex manifolds, a rich source of examples is provided by algebraic geometry, the study of solution sets of polynomial equations in $R^{n}, C^{n}$, or $C P^{n}$. We show that a supermanifold defined by a polynomial equation in the Grassmann coordinates of flat superspace is always of the DeWitt type. Thus, more general examples of the Rogers type must be sought elsewhere.

The 2-torus is a well-known example of a compact Rogers supermanifold. In fact, the 2-torus can be given several inequivalent supermanifold structures. In the simplest structure, the typical leaf of the soul foliation is an $S^{1}$ in the decomposition $S^{1} \times S^{1}$ of the torus. In another each leaf is an $R^{1}$ which is dense in the whole torus. We have found a new structure in which some leaves are compact while others are 
not. There are two compact leaves which are $S^{1}$ 's, while the other leaves are $R^{1}$ 's which are all asymptotic to the compact leaves. Like the structure in which all leaves are dense, this structure has the property that all superfields on the torus are constant. This example suggests that the full range of behavior exhibited by foliations in general can probably occur for the foliations of a supermanifold.

In our earlier paper we conjectured that a "maximal" simply connected Rogers supermanifold is necessarily a DeWitt supermanifold. Here, we discuss the appropriate sense of "maximal" for this conjecture. An example shows that the conjecture is actually false if "maximal" is interpreted in the most natural sense, and we suggest an alternative definition.

Throughout this paper, the unqualified term "supermanifold" refers to a Rogers supermanifold.

\section{Definition and Basic Properties of Supermanifolds}

This section reviews some definitions and results from [1-5] and establishes our notation.

Just as a complex manifold has coordinates whose values lie in the algebra of complex numbers, a supermanifold has coordinates with values in a Grassmann algebra. The Grassmann algebra $B_{L}$ has $L$ generators, denoted $v_{1}, v_{2}, \ldots, v_{L}$, which anticommute:

$$
v_{i} v_{j}=-v_{j} v_{i}
$$

Sequences of subscripts denote products of generators,

$$
v_{i j \ldots k} \equiv v_{i} v_{j} \ldots v_{k}, \quad i<j<\ldots<k .
$$

A general element of the algebra has the form,

$$
\begin{aligned}
A & =A_{0}+A_{i} v_{i}+A_{i j} v_{i j}+\ldots, \quad i<j<\ldots \text { in each term }, \\
& \equiv A_{\Gamma} v_{\Gamma},
\end{aligned}
$$

where the coefficients $A_{\Gamma}$, called the components of $A$, are real numbers. Note that this expansion must terminate after $L+1$ terms. In this paper we always assume $L$ is finite. The real number $A_{0}$ is called the body of $A$, while the remainder $s(A)$ $\equiv A-A_{0}$ is the soul. The element $A$ is called even (odd) when only products of even (odd) numbers of generators appear in (2.3). Odd elements mutually anticommute, even elements commute with anything, and an arbitrary element can be written as a sum of an even piece and an odd piece. The number of independent real coefficients needed to specify an even or odd element is $2^{L-1}$. Normally $x$ denotes an even element, $\theta$ an odd one, and $z$ an element of definite but unspecified parity.

Flat superspace, denoted $B_{L}^{m, n}$, is a supermanifold having $m$ even Grassmann coordinates $x^{\mu}$ and $n$ odd coordinates $\theta^{\alpha}$. It is simply the $(m+n) 2^{L-1}$-dimensional Euclidean space of the real coordinates $x_{\Gamma}^{\mu}$ and $\theta_{\Gamma}^{\alpha}$. On flat superspace there is a special class of $B_{L}$-valued functions called superfields. These are functions with polynomial dependence on the odd coordinates,

$$
F(x, \theta)=f_{0}(x)+f_{\alpha}(x) \theta^{\alpha}+f_{\alpha \beta}(x) \theta^{\alpha} \theta^{\beta}+\ldots .
$$


The coefficient functions of $x$ are also required to possess Taylor expansions in powers of $s(x)$ :

$$
f(x)=f\left(x_{0}\right)+\partial_{\mu} f\left(x_{0}\right) s\left(x^{\mu}\right)+\frac{1}{2} \partial_{\mu} \partial_{v} f\left(x_{0}\right) s\left(x^{\mu}\right) s\left(x^{v}\right)+\ldots .
$$

If the coefficient functions $f\left(x_{0}\right)$ are all analytic, the superfield is called superanalytic; if they are merely smooth the superfield is $G^{\infty}$. Note that the expansions (2.4) and (2.5) also terminate.

A supermanifold of dimension $(m, n)$ is a real manifold of dimension $(m+n) 2^{L-1}$ with the following additional structure. In each coordinate chart the real coordinates are viewed as the real components of $m x$ coordinates and $n \theta$ coordinates as in (2.3); and when charts overlap, the transition functions must be superfields. A collection of charts with these properties is called a $G^{\infty}$ (or superanalytic) structure. All supermanifolds considered in this paper will be assumed to be connected.

Through each point in a given chart of a supermanifold we may construct the surface of points with the same body coordinates $x_{0}^{\mu}$ as the chosen point. These surfaces fit together smoothly when charts overlap, giving a foliation of codimension $m$ which we call the soul foliation. Each leaf of the soul foliation itself has many subsidiary foliations defined by holding various combinations of soul coordinates fixed. For example, fixing all coordinates $z_{\Gamma}^{A}$, where $\Gamma$ is a particular sequence of subscripts, and all coordinates whose subscripts form a subsequence of $\Gamma$ gives a foliation. We call the supermanifold nice if each leaf of the soul foliation meets each chart at only finitely many distinct values of $x_{0}^{\mu}$. Of the three foliations of the 2-torus described in Sect. 1, only the foliation by $S^{1}$ 's is nice.

Given a supermanifold, we can obtain a topological quotient space by identifying all points which lie on the same leaf of the soul foliation. This space is in general not a smooth manifold, but if it is we call it the body of the supermanifold. In supergravity it serves as the physical spacetime manifold. Note that the existence of a body in this sense does not necessarily imply that the body can be embedded in the supermanifold transversely to the leaves. Conversely, given a smooth $m$-dimensional manifold $M$, there always exist $(m, n)$ supermanifolds which are vector bundles over $M$ and have body $M$, for any $n \geqq 0$. These are DeWitt supermanifolds.

The general questions which concern us are: How much more exotic can a supermanifold be than these vector bundle examples?, and, What topological conditions on a manifold determine whether it admits a $G^{\infty}$ structure?

\section{The Structure of Supermanifold Leaves}

The leaves of a DeWitt supermanifold are always vector spaces $R^{k}$. What kinds of manifolds can be leaves of a Rogers supermanifold? We will show that any leaf of the soul foliation of a supermanifold has a covering space which admits a flat Riemannian metric. Then the universal covering space of the leaf also has a flat metric, and if it is complete in this metric it is necessarily $R^{k}[6]$. This result shows that many familiar manifolds, such as spheres and projective spaces of dimension $>1$, cannot be leaves of a supermanifold. Indeed, any compact leaf must be nonsimply connected. Thus, many fiber bundles do not admit $G^{\infty}$ structures such 


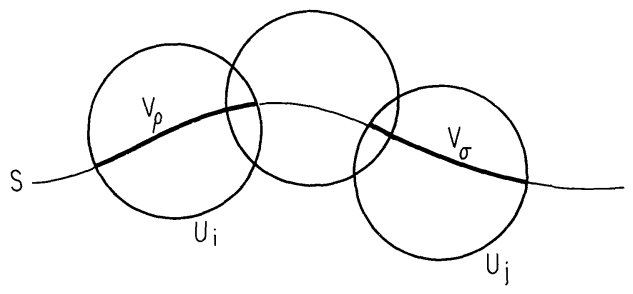

Fig. 1. Any leaf $S$ of the soul foliation of a supermanifold $M$ has a natural manifold structure. The charts on $S$ are the intersections of $S$ with the charts on $M$

that the fibers are the leaves. It would be interesting to know whether such a bundle can admit some other $G^{\infty}$ structure. Our result also holds for the leaves of the subsidiary foliations obtained by fixing any combination of coordinates $z_{\Gamma}$.

First, we observe that each leaf of the soul foliation has a natural manifold structure. A given leaf $S$ intersects a given chart $U_{j}$ of the supermanifold $M$ in a collection of hypersurfaces $x_{0}^{\mu}=$ const (Fig. 1). This collection of subsets of all the $U_{i}$ forms a system of charts $V_{\varrho}$ on $S$. The coordinate maps of $M$ send each $V_{\varrho}$ into one of the leaves of $B_{L}^{m, n}$, and each of these leaves is an $R^{k}, k=(m+n) 2^{L-1}-m$. Thus we get coordinate maps $\phi_{\varrho}: V_{\varrho} \rightarrow R^{k}$. The transition functions of this atlas on $S$ are obtained by setting $x_{0}^{\mu}=$ const in the transition functions of $M$. The transition functions of $M$ are superfields, which according to (2.4) and (2.5) are polynomial functions of all soul coordinates. Therefore we have the crucial result that for this atlas on $S$, the transition functions (and their inverses) are polynomial functions of all coordinates.

Manifolds whose transition functions are polynomials of degree 1 (linear functions) are called affine manifolds and have been extensively studied by mathematicians. In fact, it is pointed out in [5] that the leaves of some of the foliations of a supermanifold, though not the soul foliation, are affine manifolds. For example, a supermanifold has a foliation whose leaves are the surfaces of constant $z_{\Gamma}$, where $\Gamma$ runs through all sequences of subscripts which do not include the integer 1 . The transition functions for these leaves are obtained from the $G^{\infty}$ transition functions of the supermanifold by fixing these variables. These functions are necessarily polynomials of degree 1 in the remaining variables, because any term of higher degree such as $z_{\Gamma} z_{\Lambda} v_{\Gamma} v_{A}$ contains two factors $v_{1}$ and so vanishes.

It is well known that an affine manifold has a covering space with a flat metric. However, the proof [7] works for a more general class of manifolds, including all the leaves of supermanifolds. What is needed for the proof is that there must exist a group $G$ of coordinate transformations on $R^{k}$ such that each transition function of the manifold, which is defined only on an open subset of $R^{k}$, extends uniquely to one of the group elements. In our case $G$ may be taken as the group of polynomial coordinate transformations on $R^{k}$ with polynomial inverses. More specifically, it could be taken as the smaller group of all coordinate transformations which can be induced on a leaf of $B_{L}^{m, n}$ by $G^{\infty}$ transformations of $B_{L}^{m, n}$.

The strategy of the proof is to explicitly construct a covering space $\hat{S}$ of a leaf $S$, and an immersion of this space into $R^{k}$. (An immersion is a smooth map which is locally, but not necessarily globally, one-to-one.) We then define a flat metric on $\hat{S}$ 
by pulling back the flat metric of $R^{k}$ : the inner product of two tangent vectors on $\hat{S}$ is defined to be the inner product of their images in $R^{k}$. We will now sketch the proof as given in [7].

Consider the set of all ordered triples $\left(V_{\varrho}, P, g\right)$, where $P$ is a point of $S, V_{\varrho}$ is a chart containing $P$, and $g$ is an element of the group $G$. We make this set into a topological space by saying that a set of triples is open iff its set of points $P$ is open in $S$. We introduce an equivalence relation on this space by calling $\left(V_{\varrho}, P, g\right)$ and $\left(V_{\sigma}, Q, h\right)$ equivalent iff $P=Q$ and $\phi_{\sigma} \phi_{\varrho}^{-1}=h g^{-1}$. (In words, $h g^{-1}$ is the group element corresponding to a coordinate transformation relating two charts containing $P$.) The quotient space $T$ by this equivalence relation generally has many connected components; fix one and call it $\hat{S}$. $\hat{S}$ is a covering space of $S$, called the holonomy covering space, with covering map $\left[\left(V_{\varrho}, P, g\right)\right] \rightarrow P$. The desired immersion of $\hat{S}$ into $R^{k}$ is given by $\left[\left(V_{\varrho}, P, g\right)\right] \rightarrow g^{-1} \phi_{\varrho}(P)$, and this is well-defined.

This proof is so slick that we should pause to give an intuitive description of the covering space $\hat{S}$. Let us pick a base point $P_{0}$ and a chart $V_{0}$ containing $P_{0}$, and try to picture the connected component $\hat{S}$ of the quotient space $T$ containing $\left[\left(V_{0}, P_{0}, 1\right)\right]$. If $Q_{0}$ is another point in $V_{0}$, this component contains $\left[\left(V_{0}, Q_{0}, 1\right)\right]$. If $Q_{1}$ is a point in a chart $V_{1}$ which overlaps $V_{0}$, and $g_{1}$ is the group element corresponding to the coordinate transformation relating these charts, then this component contains $\left[\left(V_{1}, Q_{1}, g_{1}\right)\right]$. In fact, this component contains all $\left[\left(V_{\varrho}, Q, g\right)\right]$ such that $Q$ lies in $V_{\varrho}$ and $g$ is the group element corresponding to the cumulative coordinate transformation across all overlaps of charts encountered along some path from $P_{0}$ to $Q$. If there are closed paths from $Q$ back to $Q$ which generate different cumulative coordinate transformations, then the holonomy covering space contains as many "copies" of $Q$ as there are such different transformations. The immersion then maps each copy of $Q$ into $R^{k}$ via its cumulative coordinate transformation. This is the natural way to extend the coordinate map $\phi_{0}: V_{0} \rightarrow R^{k}$ of the initial chart to a map of the entire covering space.

Of course, if any covering space of $S$ immerses in $R^{k}$ and has a flat metric, so does the universal covering space. If the leaf $S$ is already simply connected then $S$ itself has a flat metric. This need not be true in general since the flat metric on $\hat{S}$ may not be invariant under covering transformations and so may not project down to $S$.

When these results about the leaves are combined with some known theorems about foliations of codimension one, we obtain information about the global structure of compact Rogers supermanifolds of dimension $(1, n)$. First, if the leaves are all simply connected and complete, they must be $R^{k}$ 's. It follows from Theorem 33 of [8] that the supermanifold itself is then a torus, $S^{1} \times \ldots \times S^{1}$. The situation is more complicated if the leaves are not all simply connected. According to Theorem 28 of [8], if the holonomy groups of the foliation are finite, then the supermanifold has a covering space $R \times \widetilde{S}$, where $\tilde{S}$ is either a leaf or a double cover of a leaf. (The holonomy groups of a foliation are constructed from the first homotopy groups of the leaves and measure the twisting of a leaf relative to nearby leaves. They will be finite if, for example, $\pi_{1}$ of each leaf is finite, or the foliation is nice.) If $U$ is the universal covering space of a leaf, we then know that the supermanifold has a covering space $R \times U$ which immerses in $R^{k+1}$. If the flat metric on $R \times U$ projects down to give a flat metric on the supermanifold, then its compactness implies the 
completeness of $R \times U$, so that $R \times U$ must be all of $R^{k+1}$, but this need not be true in general.

Thus, under some conditions we know that a compact Rogers supermanifold of dimension $(1, n)$ has a covering space which immerses in $R^{k+1}$, and must be all of $R^{k+1}$ if completeness is assumed. If this covering space is all of $R^{k+1}$, it has a $G^{\infty}$ structure in which the body is $R$ and the leaves are $R^{k}$ 's which are the fibers of the trivial bundle over the body. This is just the standard structure of $B_{L}^{1, n}$. If the leaves themselves have flat metrics then it seems physically reasonable to require completeness of the leaves, but if only their covers have such metrics then this requirement seems less plausible.

We have not yet exhausted the implications of the fact that the $G^{\infty}$ transition functions of a supermanifold are polynomial functions of the soul coordinates. We have shown that this yields a flatness condition on the individual leaves of the foliations, but it also restricts the way in which the leaves can twist around each other. Fix a leaf $S$ of the soul foliation and an immersion of its universal covering space $U$ into $R^{k}, k=2^{L-1}(m+n)-m$. The manifold $S$ is itself foliated by leaves along which various combinations of soul coordinates are held fixed, and there are corresponding foliations of $U$. Let us consider one such subsidiary foliation whose leaves are locally defined by holding fixed some set of soul coordinates $z_{\Gamma}^{A}, q$ in number. By composing the immersion $U \rightarrow R^{k}$ with the projection $R^{k} \rightarrow R^{q}$ onto the coordinates $z_{\Gamma}^{A}$, we get a submersion $\pi: U \rightarrow R^{q}$. Because of the way the immersion was constructed, the leaves of the subsidiary foliation of $U$ are precisely the level sets of $\pi ; \pi$ maps the leaves of $U$ to points in $R^{q}$.

Foliations whose leaves are the level sets of a submersion are much better behaved than foliations in general. For example, the leaves are nice. In fact, a given leaf can cut a given chart at only one value of the transverse coordinates $z_{\Gamma}^{A}$, by definition. In particular, the leaves cannot be dense. It also follows that the holonomy groups of the leaves are trivial because $U$ is simply connected [9]. The point of this analysis is that most of the complexity of the foliation structure of a supermanifold is associated with the soul foliation, for which we have no defining submersion unless the supermanifold has a body. The subsidiary foliations are much nicer and less structure is possible for them.

\section{Algebraic Geometry in Superspace}

As mentioned in the Introduction, and as we can personally testify, it is quite difficult to find examples of supermanifolds other than vector bundles and their subsets and quotient spaces. We do not yet know whether this reflects a general structure theorem for supermanifolds or our own lack of creativity. For real and complex manifolds, a rich source of examples is provided by algebraic geometry, the study of polynomial equations in $R^{m}, C^{m}$, or $C P^{m}$. This suggests that one study supermanifolds defined by polynomial equations in $B_{L}^{m, n}$. Unfortunately, all supermanifolds of this type are vector bundles. This can be shown explicitly when the polynomial has real coefficients, by constructing the base space (body) and the vector bundle charts. We then treat arbitrary Grassmann coefficients as perturbations of real ones and show that such perturbations produce a diffeomorphic manifold. 
Consider the set of points in $B_{L}^{m, n}$ which satisfy the polynomial equation,

$$
P(a, x, \theta)=0,
$$

where $a$ stands for the coefficients of the polynomial, which may be arbitrary elements of $B_{L}$, and indices have been suppressed. In physics one normally assumes that all superfields take values in the even sector of $B_{L}$ only, so we shall initially consider only even polynomials $P$. We also assume at first that the coefficients $a$ are all real numbers; $s(a)=0$. A typical example of a polynomial of this type would be

$$
\left(x^{1}\right)^{2}+\left(x^{2}\right)^{2}+\left(x^{3}\right)^{2}+\theta^{1} \theta^{2}-1=0,
$$

which defines a "supersphere" in $B_{L}^{3,2}$. Assuming that such a polynomial defines a supermanifold, each point must have a neighborhood in which it is possible to solve for one of the coordinates in terms of the remaining ones. (Alternatively, one can simply delete the points at which this is not possible and study the remaining set.) However, it is never possible to solve an even polynomial for a $\theta$ coordinate, because the coefficient of any $\theta$ coordinate must be an odd expression. Given any particular solution, an infinite number of others are found by shifting any $\theta$ coordinate by an amount proportional to its odd coefficient. In (4.2), for example, $\theta^{1}$ could be shifted by a multiple of $\theta^{2}$. Thus, an even polynomial never uniquely determines any $\theta$ variable in terms of the others. An even polynomial in $B_{L}^{m, n}$ therefore defines a supermanifold, which must have dimension $(m-1, n)$, iff every point has a neighborhood in which it is possible to solve for one of the $x$ coordinates in terms of the other variables. According to the $G^{\infty}$ implicit function theorem [10], one can solve for $x^{\mu}$ in any neighborhood in which $\partial P / \partial x^{\mu}$ is invertible (has nonzero body), and $x^{\mu}$ will be a $G^{\infty}$ function of the remaining variables $x^{\nu}, \theta^{\alpha}$, and the coefficients $a$.

Notice now that the body coordinates of any point obeying (4.1) will satisfy

$$
P\left(a_{0}, x_{0}, 0\right)=0 \text {. }
$$

In fact, this equation defines a submanifold of (4.1) which is just the intersection of (4.1) with the body $R^{m}$ of $B_{L}^{m, n}$. We call this submanifold the body of (4.1). For the supersphere (4.2), for example, it is an ordinary sphere in $R^{3}$. We now claim that (4.1) has the structure of a vector bundle over its body. Any point of the body has a neighborhood in the supermanifold in which one can solve for some $x^{\mu}$ as a $G^{\infty}$ function of the other coordinates. Such a function has polynomial dependence on all soul coordinates, so its radius of convergence with respect to these coordinates is infinite. Thus, the neighborhood in which one can solve for $x^{\mu}$ can be taken to be an entire cylinder over an open set in the body. A covering of the supermanifold by such neighborhoods gives it the required vector bundle structure. Notice that the $\theta$ coordinates are global coordinates on the entire supermanifold, so this bundle has a large trivial subbundle. We investigated the structure of such bundles in [4] and showed them to be direct sums of trivial bundles and copies of the tangent bundle to the body.

Now we generalize the discussion to even polynomials $P$ with arbitrary Grassmann coefficients $a$. The essential difference here is that (4.3) is no longer a submanifold of (4.1), even though the body coordinates do satisfy (4.3). We will 
prove that replacing the coefficients of the polynomial by their bodies gives a diffeomorphic supermanifold, which we have just shown to be a vector bundle. We do this by splitting each coefficient into body and soul, and treating the soul terms as perturbations.

First, we review the theory of perturbations of real polynomial equations. Assume that $P(a, x)=0$ is a polynomial equation with real variables and coefficients which defines a manifold in $R^{m}$. Let

$$
P(a, \varepsilon, x)=P(a, x)+\varepsilon Q(x)=0
$$

be the perturbed equation, with $\varepsilon=0$ corresponding to the unperturbed polynomial. We wish to know when the equation with $\varepsilon=1$ defines a manifold diffeomorphic to the unperturbed one.

Let us try to construct a diffeomorphism between the solution sets of these equations. Consider all the points of $R^{m}$ which satisfy one of the Eqs. (4.4) for some $\varepsilon$. At each such point we construct the normal vector $\nabla P$ to the polynomial it satisfies. This defines a vector field except at points where $P(a, x)=Q(x)=0$, which satisfy (4.4) for all values of $\varepsilon$. If this vector field vanishes at any point of (4.4) for $0 \leqq \varepsilon \leqq 1$, then (4.4) does not define a manifold at this value of $\varepsilon$ and we do not expect to get a diffeomorphism. If, on the other hand, the gradient vector field is nonvanishing, then its integral curves are normal to the manifolds (4.4). Provided that each integral curve meets both manifolds $\varepsilon=0,1$ the relation of lying on the same curve sets up a one-to-one smooth correspondence between distinct points of these manifolds, showing that they are diffeomorphic. (The points which lie on all the manifolds are simply left fixed by the diffeomorphism.) A simple example where this does not work is provided by the curves

$$
x^{2}+\varepsilon y^{2}=1
$$

in $R^{2}$. For $\varepsilon=0$ this is a pair of lines parallel to the $y$ axis, while for $\varepsilon=1$ it is a circle. These manifolds are not diffeomorphic; the problem is that the integral curves through the points $(0, \pm 1)$ of the circle are parallel to the lines and never meet them. To see that the Grassmann case is different, the reader should check that in $B_{L}^{2,0}(4.5)$ represents a pair of parallel hyperplanes for all pure soul values of $\varepsilon$.

Returning to the situation of interest, we want to show that the equations $P(a, x, \theta)=0$ and $P\left(a_{0}, x, \theta\right)=0$ represent diffeomorphic manifolds in $B_{L}^{m, n}$. First, both these equations do in fact represent supermanifolds if either does, because this depends only on the bodies of the coefficients. Next, writing $P=P_{\Gamma} v_{\Gamma}$ with real polynomials $P_{\Gamma}$, we write out the equations $P_{\Gamma}(a, x, \theta)=0$. They will take the form,

$$
\begin{aligned}
& \Gamma=0: P\left(a_{0}, x_{0}, 0\right)=0, \\
& \Gamma=i j: P_{i j}\left(a_{0}, x_{0}, x_{i j}, \theta\right)=-Q\left[s(a), x_{0}, \theta\right] . \\
& \ldots
\end{aligned}
$$

The equations are written out in such a way that the perturbation terms involving $s(a)$ appear on the right; the unperturbed equations would have zero on the right. These equations have the following crucial property. The variables $x_{\Gamma}$ first appear in the equation labeled $\Gamma$, and they appear linearly and on the left side only. 
We will now construct the correspondence (diffeomorphism) between unperturbed points obeying (4.6) with zeroes on the right, and perturbed points obeying (4.6) itself. Since the first equation in (4.6) does have zero on the right, and since the $\theta$ coordinates are always global coordinates on supermanifolds of this type, we declare that the diffeomorphism is not to change the $\theta$ and $x_{0}$ coordinates of each point. The unknowns in the second of Eqs. (4.6) are now the $x_{i j}$. Since they appear linearly, the gradient with respect to these variables is constant in magnitude and direction. Therefore, by flowing along the gradient for a finite distance we can reach points for which the right side of the equation takes any finite values. The integral curves, which are just straight lines, do therefore meet both the perturbed and unperturbed manifolds, and from a given unperturbed point we can find the $x_{i j}$ coordinates of the corresponding perturbed point and vice versa. The next equation of (4.6) then fixes the $x_{i j k l}$ coordinates of corresponding points, and the diffeomorphism is inductively constructed.

We would like to give an alternative argument for the existence of this diffeomorphism which is more intuitive and does not require decomposing the polynomial into components in a particular basis of $B_{L}$. We have been treating the equation $P(a, x, \theta)=0$ as a perturbation of $P\left(a_{0}, x, \theta\right)=0$. Let us formally treat $x$ as real variables and $\theta$ as real constants and consider real perturbations of this equation with real parameter $\varepsilon$. In a neighborhood of any particular unperturbed solution we can always choose $\varepsilon$ so small that a perturbation series in $\varepsilon$ converges and gives a local diffeomorphism of the neighborhood onto a neighborhood of a perturbed solution. The problem is that different neighborhoods may require different values of $\varepsilon$, so that no value gives a perturbation series which converges globally. However, if $\varepsilon$ is reinterpreted as a pure soul Grassmann parameter, all the local perturbation series will have infinite radius of convergence because they will terminate. There is then no difficulty in constructing the diffeomorphism globally.

The situation for odd polynomials $P$ is similar. Each power of $x^{\mu}$ appears with an odd coefficient in such a polynomial, so $\partial P / \partial x^{\mu}$ cannot be invertible at any point and at best such a polynomial can only be solved for $\theta$ coordinates. Furthermore, the polynomial is necessarily linear in each $\theta^{\alpha}$, so it can be solved for any $\theta^{\alpha}$ whose coefficient has nonzero body. We then get an $(m, n-1)$ supermanifold on which the $x$ 's are global coordinates. It will be a vector bundle over some region in the body $R^{m}$ of $B_{L}^{m, n}$. In contrast to the even case, pure soul perturbations of the coefficients cannot change this region at all here. This region need not be all of $R^{m}$ because it may not be possible to solve for any $\theta$ coordinate at some values of $x_{0}$. For example, the equation $(1-x) \theta=v_{1}$ cannot be solved for $\theta$ if $x_{0}=1$.

Finally, a polynomial which is neither even nor odd can always be split into even and odd parts. The even part defines a vector bundle over an $(m-1)$-manifold $B$ in $B_{L}^{m, n}$, and the odd part defines a vector bundle over a region $R$ of $R^{m}$. The fibers of each bundle are subspaces of the soul fibers of $B_{L}^{m, n}$. The polynomial itself defines a supermanifold of dimension $(m-1, n-1)$ which is a vector bundle over the set of points of $B$ whose bodies lie in $R$.

We expect similar results to hold for supermanifolds defined by several simultaneous polynomial equations. All these supermanifolds are of the DeWitt type. 


\section{A New Supermanifold Structure on the 2-Torus}

As for complex manifolds, an interesting question is the number of distinct $G^{\infty}$ structures a given real manifold may admit. In [4] we reviewed the two known types of $G^{\infty}$ structure on the torus. One structure is obtained by taking the quotient space of $B_{2}^{1,0}$ by the group of integer translations in the $x_{0}$ and $x_{12}$ directions. The leaves of the soul foliation are circles, there is a body which is also a circle, and superfields must be constant along leaves but need not be globally constant. A different structure is obtained by taking the quotient space of $B_{2}^{1,0}$ by integer translations along two perpendicular axes which make a suitable angle with the $x_{0}$ and $x_{12}$ axes. Here the leaves are irrational dense torus knots, there is no body, and superfields must be globally constant. We indicated that all supermanifolds known to us were either nice or had dense leaves.

We will now present a new $G^{\infty}$ structure on the 2-torus which is not nice, yet has no dense leaves. The previous sections of this paper have stressed similarities between the supermanifolds of DeWitt and Rogers; this section emphasizes that Rogers supermanifolds have greater variety despite the previous results.

Consider the region in $B_{2}^{1,0}$ shown in Fig. 2. It is bounded by the line $x_{12}=0$ and the graph of $x_{12}=3+\frac{1}{2} \cos x_{0}$, and is divided into three subregions by the lines $x_{12}=1$ and $x_{12}=2$. Each point $x$ in region $A$ is now identified with the point $y$ in region $B$ given by the invertible superanalytic map,

$$
y=x+\frac{1}{2} \sin x+2 v_{12},
$$

which appears in components as

$$
\begin{gathered}
y_{0}=x_{0}+\frac{1}{2} \sin x_{0}, \\
y_{12}=x_{12}\left(1+\frac{1}{2} \cos x_{0}\right)+2 .
\end{gathered}
$$

This gives a superanalytic structure on a cylinder $R^{1} \times S^{1}$. The corresponding structure on the torus then follows by identifying points related by translations along $x_{0}$ by $2 n \pi$.

The leaves of $B_{2}^{1,0}$ are of course just the lines parallel to the $x_{12}$ axis. Each leaf is labeled by a value of $x_{0}$. The function (5.2), graphed in Fig. 3, tells which leaf in

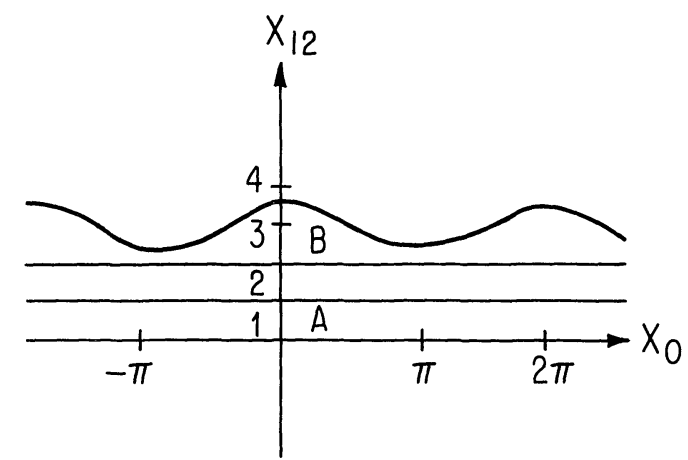

Fig. 2. The region in $B_{2}^{1,0}$ from which the torus is constructed by identifying subregions $A$ and $B$. Subregion $A$ is bounded by the lines $x_{12}=0$ and $x_{12}=1$, and subregion $B$ is bounded by $x_{12}=2$ and the graph of $x_{12}=3+\frac{1}{2} \cos x_{0}$ 


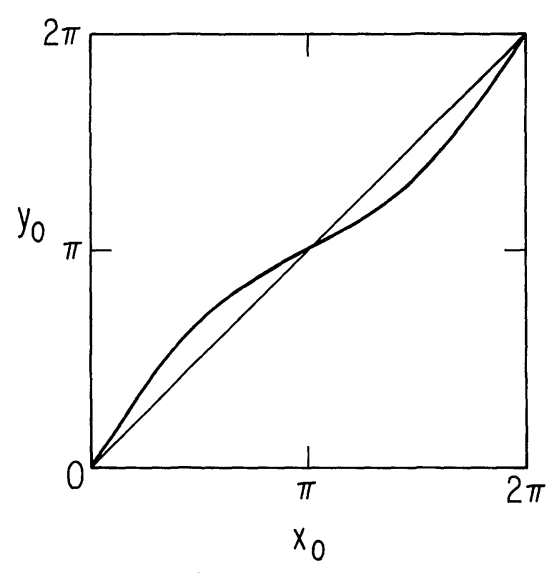

Fig. 3. The graph of Eq. (5.2), $y_{0}=x_{0}+\frac{1}{2} \sin x_{0}$. The leaf $x_{0}$ in region $A$ is identified with the leaf $y_{0}$ in region $B$. The line $y_{0}=x_{0}$ is also shown to locate the fixed points

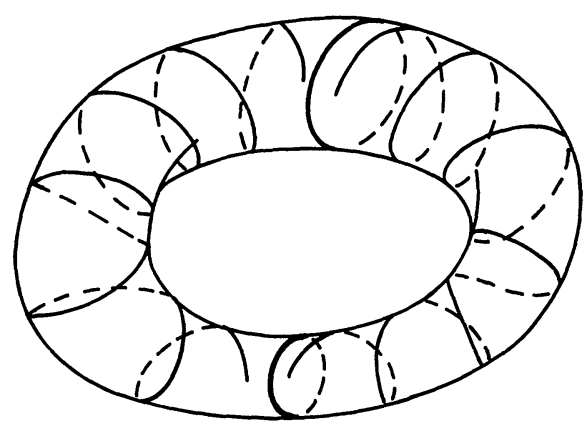

Fig. 4. Leaves of the new superanalytic structure on the torus. All other leaves are asymptotic to the two circular ones

region $B$ is identified with a given leaf in region $A$ under (5.1). This function has a stable fixed point at $x_{0}=\pi$ and an unstable one at $x_{0}=0$. This implies that the leaves $x_{0}=0$ and $x_{0}=\pi$ on the torus are circles. Every other leaf has the topology of $R^{1}$ and is asymptotic to the circle $x_{0}=0$ in the positive $x_{12}$ direction and to the other circle $x_{0}=\pi$ in the negative $x_{12}$ direction. This is shown in Fig. 4. This example shows that the leaves of a Rogers supermanifold need not all be diffeomorphic.

In [4] we established some results on superfields on Rogers supermanifolds. We showed that if all leaves are compact, a superfield is necessarily constant along each leaf. We have recently found a simpler argument which actually establishes that superfields are constant on any particular compact leaf. Write a global superfield as $F=F_{\Gamma} v_{\Gamma}$ with real-valued global functions $F_{\Gamma}$. The restrictions of the $F_{\Gamma}$ to the compact leaf are real-valued polynomial functions of the soul coordinates along the leaf, which moreover are first-degree in each real variable separately. By compactness, they achieve maximum and minimum values on the leaf. But elementary methods show that such a function cannot have any local extrema unless it is constant. 
In principle, if some leaves are compact and others are not, there can be superfields which are constant only on the compact leaves. Unfortunately, the structure we are discussing on the torus does not have this property. Consider a superfield $F(x)=F_{\Gamma}(x) v_{\Gamma}$ on the torus, and recall [4] that its leading terms (the terms containing the smallest number of generators $v_{j}$ ) are constant along all leaves. Since every leaf is asymptotic to the circular leaves, the leading terms take the same value on every leaf that they do on either circular leaf. But this makes the leading terms globally constant. If this global constant is subtracted from $F(x)$, the same argument applies to the new leading terms. Hence all superfields on the torus are globally constant. We suspect that one must go to supermanifolds with several even dimensions, so that the soul foliation has higher codimension, to get an interesting example with nonconstant superfields.

\section{Conclusions}

In this section we summarize our work and indicate some important open problems, including our conjecture on the global structure of Rogers supermanifolds.

We have shown that a general Rogers supermanifold retains much of the structure of a DeWitt supermanifold. The leaves of the soul foliation have flat covering spaces, which are vector spaces if they are complete. The subsidiary foliations of these flat covering spaces are defined by submersions. Supermanifolds defined by polynomial equations in flat superspace are always vector bundles over their bodies, but Rogers supermanifolds exhibit considerably more variety than these examples. Nevertheless, all our examples are consistent with our conjecture that a "maximal" simply connected Rogers supermanifold is always a vector bundle over a body.

The most natural sense of "maximal" for this conjecture is the following. A supermanifold $M$ is maximal iff there does not exist a $G^{\infty}$ embedding of $M$ into a supermanifold $N$ of the same dimension which takes some leaf of $M$ into a proper subset of a leaf of $N$. This definition is attractive because the conjecture then says that the universal covering space of an arbitrary supermanifold can be realized as an open subset of a DeWitt supermanifold. However, this definition makes the conjecture false, as the following example shows. Let $K$ be the supermanifold obtained from $B_{2}^{1,0}$ by deleting the origin. Note that this deletion splits the leaf $x_{0}=0$ into two leaves. These two leaves are not complete in the flat metrics associated to them by the construction of Sect. 3. This is reflected in the properties of superfields: there exist superfields which are unbounded in only one direction along these leaves. Also, once the origin is deleted there is no longer an embedded body manifold transverse to the leaves and meeting each leaf exactly once. Now let $M$ be the universal covering space of $K$, with the induced $G^{\infty}$ structure. Introducing polar coordinates in $B_{2}^{1,0}, M$ is just the set of points $(\theta, r)$ where $r>0$ and $\theta$ is not identified with $\theta+2 \pi$. If we take as new coordinates $(\theta, \log r), M$ becomes $R^{2}$ with a nonstandard $G^{\infty}$ structure. The original leaves $x_{0}=a$ of $B_{2}^{1,0}$ are covered by the curves $r \cos \theta=a$ in $M$. In particular, the leaf $x_{0}=0$, from which the origin was deleted, is covered by the leaves $\theta=\left(n+\frac{1}{2}\right) \pi$, and these leaves are incomplete in their natural flat metrics. The leaves of the new $G^{\infty}$ structure are 


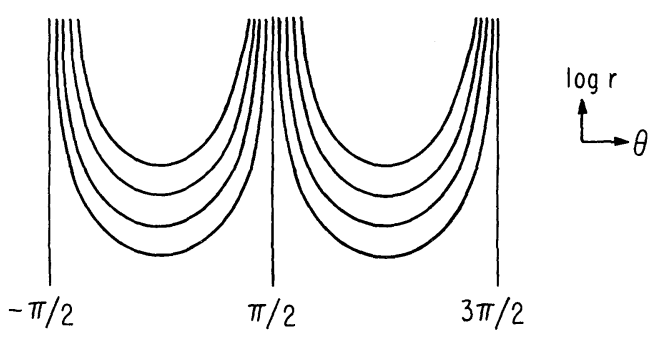

Fig. 5. Leaves of the new superanalytic structure on the plane $M$. The curves are all congruent and are all asymptotic to the vertical lines. Clearly there exists no line (body) transverse to the leaves and intersecting each leaf exactly once

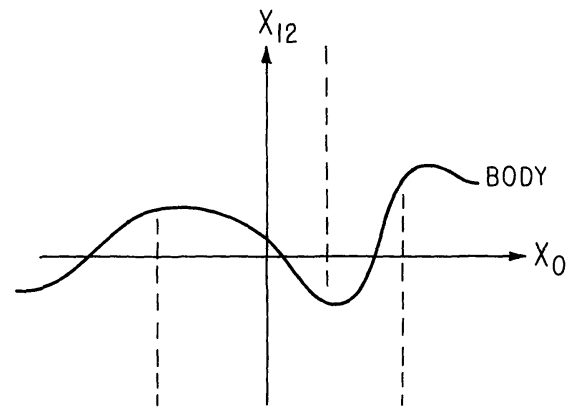

Fig. 6. A candidate for a realization of the supermanifold $M$ as an open subset of $B_{2}^{1,0}$. The dashed lines are deleted from the leaves. A body manifold is shown

shown in Fig. 5. There is still no embedded body manifold. Contrary to the conjecture, there is no $G^{\infty}$ map of $M$ into the vector bundle $B_{2}^{1,0}$ taking leaves to leaves. If there were, it would have to send the incomplete leaves of $M$ into proper subsets of leaves of $B_{2}^{1,0}$ and all other leaves of $M$ into entire leaves. Thus, the image of $M$ in $B_{2}^{1,0}$ would be the open subset obtained by making cuts along a discrete set of leaves as in Fig. 6. These cuts must go out to infinity in one direction since otherwise the image would not be simply connected. But it is clear that in Fig. 6, there is a body manifold meeting each leaf once, so this is not a faithful realization of the $G^{\infty}$ structure of $M$.

A stronger sense of "maximal" is that the universal covering spaces of all leaves of $M$ should be complete in their flat metrics. This eliminates the above counterexample, but is not well motivated physically. Also, with this definition the conjecture gives no information about the structure of nonmaximal supermanifolds because they cannot necessarily be realized as subsets of maximal ones. However, we still believe it is important to either prove the conjecture or produce a counterexample.

Many interesting questions about supermanifolds remain open. The set of known examples of supermanifolds is still too small to give a clear idea of the range of possibilities. In particular, we would like to see an example in which there are superfields constant on some, but not all, leaves. Examples of this type are the only 
compact supermanifolds which can be useful in physics: a superfield which is constant on all leaves cannot transform under supersymmetry and cannot represent a supermultiplet of ordinary fields. There are also constraints on $G^{\infty}$ differential forms on supermanifolds having compact leaves which have yet to be worked out. We do not know how many distinct $G^{\infty}$ structures simple manifolds like $R^{n}$ and $T^{n}$ possess. Finally, it is still not clear whether a notion of Berezin integration can be defined on supermanifolds more general than those of DeWitt. This problem will be considered in a forthcoming paper [11].

Acknowledgements. We thank R. Blattner, R. Geroch, M. Hirsch, and M. Rothenberg for helpful conversations.

\section{References}

1. Rogers, A.: A global theory of supermanifolds. J. Math. Phys. 21, 1352-1365 (1980)

2. DeWitt, B.S.: Supermanifolds. Cambridge: Cambridge University Press 1984

3. Hoyos, J., Quirós, M., Mittelbrunn, J.R., de Urriés, F.J.: Generalized supermanifolds. III. Q-supermanifolds. J. Math. Phys. 25, 847-854 (1984)

4. Rabin, J.M., Crane, L.: Global properties of supermanifolds. Commun. Math. Phys. 100, 141-160 (1985)

5. Boyer, C.P., Gitler, S.: The theory of $G^{\infty}$-supermanifolds. Trans. Am. Math. Soc. 285, 241-267 (1984)

6. See, for example, Kobayashi, S., Nomizu, K.: Foundations of differential geometry, Vol. I, p. 265. New York: Interscience 1963

7. Nagano, T., Yagi, K.: The affine structures on the real two-torus (I). Osaka J. Math. 11, 181-210 (1974)

8. Lawson, H.B.: Foliations. Bull. Am. Math. Soc. 80, 369-418 (1974) and references therein

9. Blumenthal, R.A.: Transversely homogeneous foliations. Ann. Inst. Fourier, Grenoble 29, 143-158 (1979)

10. Hoyos, J., Quirós, M., Mittelbrunn, J.R., de Urriés, F.J.: Generalized superspaces. II. Analysis on superspaces. J. Math. Phys. 25, 841-846 (1984); Jadczyk, A., Pilch, K.: Superspaces and supersymmetries. Commun. Math. Phys. 78, 373-390 (1981)

11. Rabin, J.M.: Berezin integration on general fermionic supermanifolds. University of Chicago preprint EFI 85-33. Commun. Math. Phys. (to appear)

Communicated by S.-T. Yau

Received May 10, 1985 
